\title{
Revisión
}

\section{LAS ZOONOSIS TRANSMITIDAS POR ALIMENTOS PUEDEN AFECTAR SU EPIDEMIOLOGÍA, PRODUCTO DEL CAMBIO CLIMÁTICO Y LOS PROCESOS DE GLOBALIZACIÓN}

\author{
Review \\ ZOONOSIS TRANSMITTED BY FOOD CAN AFFECT ITS \\ EPIDEMIOLOGY, AS A RESULT OF CLIMATE CHANGE AND PROCESSES \\ OF GLOBALIZATION
}

\author{
Sergio Donoso ${ }^{1^{*}}$, Paula Gadicke ${ }^{1}$, y Carlos Landaeta ${ }^{1}$ \\ ${ }^{1}$ Departamento de Patología y Medicina Preventiva, Facultad de Ciencias Veterinarias, Universidad de \\ Concepción, Casilla 537, Chillán, Chile. \\ * Autor para correspondencia E-mail: sedonoso@udec.cl
}

\section{RESUMEN}

El propósito de esta revisión es evaluar los efectos del cambio climático y de la globalización en el comportamiento epidemiológico de las zoonosis transmitidas por los alimentos. Los mayores efectos se han generado por la globalización, donde el cambio de hábitos de las personas, el aumento en el transporte de pasajeros, el movimiento de animales, la mayor disponibilidad de alimentos, y el aumento en el comercio internacional de alimentos, ingredientes y materias primas, han generado mayor diseminación de agentes patógenos. Las modificaciones de las temperaturas y el aumento en la frecuencia de fenómenos climáticos extremos, como grandes sequías e inundaciones, han afectado a los patógenos que se pueden transmitir por los alimentos, lo que ha generado la desaparición y aparición de nuevos patógenos en los alimentos. Debido a que el cambio climático y la globalización son procesos constantes y dinámicos, se necesita mayor investigación y colaboración entre las organizaciones mundiales relacionadas con los alimentos, con la sanidad humana y animal, que permitan determinar el efecto que estos fenómenos tienen sobre las zoonosis transmitidas por los alimentos.

Palabras clave: zoonosis, globalización, cambio climático, alimentos, enfermedades.

\section{ABSTRACT}

The objective of this review was to evaluate the effects of climate change and globalization on the epidemiological behavior of zoonosis transmitted by food. Globalization has affected people's habits and has led to an increased transport of passengers and movement of animals, greater availability of foods and an increase in the international trade of foods, ingredients and raw materials, resulting in an increased spread of pathological agents. The changes in temperatures and the increased frequency of extreme climatological events, such as severe droughts and flooding, have affected pathogens that can be transmitted by food, leading to the disappearance of old pathogens and emergence of new pathogens in foods. Since climate change and globalization are constant and dynamic processes, further research and support from world organizations for food and human and animal health are needed to determine the effect of these phenomena on zoonosis transmitted by foods.

Key words: zoonosis, globalization, climate change, foods, diseases 


\section{INTRODUCCIÓN}

El planeta está permanentemente sufriendo cambios que están afectando el clima, el comercio, los hábitos y los movimientos de las personas y las comunicaciones. Estos cambios repercuten directamente en la población humana y animal que habita el planeta. La población está pasando de la territorialidad a la extraterritorialidad, de la seguridad a la inseguridad y de la inmovilidad a la movilidad en un proceso dicotómico que ha estado y seguirá alterando la vida en el planeta (Tirado et al., 2010).

El gran crecimiento de la población mundial ha generado una creciente demanda por alimentos con la finalidad de satisfacer las necesidades alimenticias. Estos alimentos deben y tienen que ser seguros para la población. Esto implica que su consumo no debe generar ningún tipo de patología ya sea de origen bacteriano, viral, parasitario, toxicológico, fúngico y priónico. Es decir, se debe tener un alimento inocuo para el consumo, y que a la vez debe estar disponible para toda la población, lo que se denomina seguridad alimentaria.

La globalización es el resultado de la innovación humana y del progreso tecnológico, corresponde a un proceso dinámico de tipo cultural, social, y económico, a gran escala, que se traduce en la creciente comunicación e interdependencia entre diversas regiones y países del mundo, generándose uniones en las culturas, sociedades y mercados. Esto ha traído un sinnúmero de consecuencias tanto positivas como negativas. Entre las positivas destacan el crecimiento económico, los avances sociales y el mejoramiento en la calidad de vida. Entre los aspectos negativos destacan el aumento en la contaminación, desigualdad, marginación, monopolios, inmigración y pobreza (Robertson et al., 2014).

La alimentación no ha quedado al margen de los procesos de globalización, pues ha permitido disponer de una gran cantidad de productos nuevos, que se han ido incorporando a la dieta de las personas. Sin embargo, ha traído también como consecuencias el ingreso al país de nuevos agentes patógenos que se han vehiculizado por los alimentos, y que pueden representar un serio riesgo para la población que los consume, generando cuadros de enfermedades transmitidas por los alimentos. A esto se ha sumado el cambio climático, donde el calentamiento global ha producido modificaciones en los patrones de comportamiento de diversos agentes patógenos, lo que ha afectado tanto su patogenicidad como su virulencia, generando cambios en las enfermedades que ellos producen y en los tratamientos utilizados (Tirado et al., 2010).

También es necesario destacar los cambios que se han ido generando en la población mundial, la que se encuentra más expuesta a los agentes patógenos priónicos, virales, bacterianos y micóticos, con consecuencias sobre la salud humana, produciendo cuadros de deficiencias inmunológicas que junto a los contaminantes presentes en el ambiente, provocan un aumento en la susceptibilidad de la población a agentes patógenos vehiculizados por los alimentos (Friedman et al., 2014).

Desde los años 50 se han generado investigaciones en donde se intenta determinar las consecuencias que ambos procesos, cambio climático y globalización, han tenido sobre la población humana, en especial sobre las modificaciones generadas en las enfermedades zoonóticas que se transmiten a través de los alimentos (Polley et al., 2011).

Los resultados de estas primeras investigaciones evidencian preliminarmente que el riesgo de contraer enfermedades zoonóticas a través de los alimentos ha aumentado, con graves consecuencias para la salud humana, para la economía de un país y para la sociedad, sin embargo, concluyen además, que se necesita mayor cantidad de recursos y de estudios, entre ellos, recopilación y análisis de datos que permitan definir fehacientemente los efectos generados por estos cambios, en un periodo de tiempo más extenso.

El desarrollo en las comunicaciones, la creación de grupos de investigación y la colaboración con organismos internacionales relacionados con la salud humana, salud animal y alimentación, permiten a la fecha disponer de una amplia gama de investigaciones y publicaciones que tratan de evidenciar las consecuencias de estos cambios en las enfermedades alimentarias (Tirado et al., 2010; Robertson et al., 2014).

Debido a los antecedentes mencionados, el objetivo de ésta revisión bibliográfica fue reunir información actualizada para evaluar los efectos de la globalización y del cambio climático en la presentación de las zoonosis transmitidas por los alimentos.

\section{MATERIALES Y MÉTODOS}

Se revisaron las fuentes bibliográficas contenidas en la Biblioteca de la Universidad de Concepción, campus Chillán, además, de diversos gestores de búsqueda bibliográficas como: Pubmed, ScienceDirect, SciELO, Web of Science y SCOPUS, además de, las bases de datos de la Organización Mundial de la Salud (OMS) y de la Oficina Internacional de Epizootias (OIE). Se utilizaron las palabras clave de búsqueda: zoonosis, alimentos, globalización, cambio climático, se seleccionaron publicaciones en idioma español e inglés, de preferencia de los últimos 10 años, 
consideradas relevantes para la realización de las presente revisión bibliográfica.

\section{RESULTADOS}

La Organización Mundial de la Salud (OMS, 2007) y Broglia y Kapel (2011), mencionan la existencia de múltiples factores por los cuales se ha apreciado un aumento en la presencia de las enfermedades zoonóticas, incluso aquellas que se creían controladas, y que están contribuyendo al incremento de las tasas de presentación de las enfermedades transmitidas por alimentos (ETAs). Dentro de estos factores se incluyen cambios en: las prácticas agrícolas y en los sistemas de producción animal; el comercio, manipulación, procesamiento y transporte de los alimentos; también se incluyen el calentamiento global y el cambio climático; mayor proporción de individuos inmunológicamente comprometidos; rápido crecimiento de la población; aparición de nuevos agentes infecciosos y cambios genéticos (mutaciones) de los existentes; reorientación de políticas de salud para evitar las enfermedades infecciosas; resistencia de los diversos agentes patógenos a las drogas utilizadas para su control; mercados cada vez más globales; cambios en los hábitos alimenticios; escaso desarrollo económico de algunas regiones, y una población humana cada vez más migratoria (OMS, 2007).

\section{Cambio climático}

El cambio climático no solo ha alterado los sistemas de producción de alimentos, la cadena de suministros y la seguridad alimentaria (Gregory et al., 2005), sino también la incidencia y prevalencia de las ETAs (Tirado et al., 2010), afectando los patrones de ocurrencia de muchas de las enfermedades presentes en las personas, en los animales domésticos y en los animales de vida libre (Polley et al., 2011), junto con las condiciones de vida de la mayoría de las especies que habitan la tierra (Uyttendaele et al., 2015). De este modo se han modificado los ciclos de vida, los mecanismos de transmisión y la patogenicidad de muchos agentes infecciosos, incluyendo los que causan enfermedades en los seres humanos (Altizer et al., 2013).

Se han observado modificaciones en la temperatura, las precipitaciones, humedad relativa, la radiación ultravioleta y aumento de las emisiones de gases con efecto invernadero, como el dióxido de carbono (IPPC, 2014, citado por Roberts et al., 2014). Sobre la base de investigaciones realizadas, se espera a futuro un clima con temperaturas más altas y extremas (Millán, 2014), cambios que van a afectar la biología y ecología de los patógenos (Marques et al., 2010) y la distribución de las enfermedades infecciosas (Altizer et al., 2013). Los fenómenos meteorológicos extremos, como inundaciones y sequías, pueden provocar la contaminación de los suelos, de las tierras agrícolas, del agua y de los alimentos, con patógenos provenientes de humanos y animales (Tirado et al., 2010). El calentamiento, la acidificación y los cambios en la salinidad de los océanos y de las precipitaciones, afectan las propiedades bioquímicas del agua, su microflora, la fisiología de los peces, la persistencia y patrones de ocurrencia de vibrios patógenos, y la aparición de algas nocivas contaminantes de las aguas y de los animales marinos (Uyttendaele et al., 2015).

Además de los efectos directos sobre la presentación de las ETAs, el cambio climático puede afectar la producción de cultivos y la sanidad vegetal, la producción y sanidad animal, las etapas de fabricación y procesamiento de alimentos, y la calidad sanitaria de los alimentos disponibles para los consumidores (Broglia y Kapel, 2011). Estos a su vez impactan en la salud pública, con consecuencias económicas, sociales y medioambientales (Tirado et al., 2010).

Respecto del calentamiento global, un estudio en Europa mostró que los casos de salmonelosis se incrementaron en $5,1 \%$ por cada grado de aumento de la temperatura ambiental semanal, para temperaturas sobre $5^{\circ} \mathrm{C}$, lo que sugiere que una inadecuada temperatura de almacenamiento y manejo de los alimentos pueden ser factores importantes en la transmisión (Kovats et al., 2005); en el caso de Irlanda se espera un aumento de alrededor del $2 \%$ en la incidencia de salmonelosis en las próximas décadas, asociados al calentamiento global (Cullen, 2009). Algo similar sucede con las vibriosis, observándose en los últimos 15 años un incremento significativo de casos por $\mathrm{Vi}$ brio parahaemolyticus, pasando de brotes esporádicos a grandes brotes atribuidos al consumo de ostras recolectadas en aguas con mayor temperatura (Drake et al., 2007). Vibrio cholerae es el mejor modelo para entender el potencial de los cambios inducidos por el clima en la transmisión de este patógeno, ya que la enfermedad es endémica sólo en ciertas regiones del mundo: zonas tropicales y subtropicales. Hashizume et al. (2009), demostraron a través de los estudios realizados, que la variación en el número de pacientes con cólera en Bangladesh podría explicarse por el aumento en las temperaturas de las aguas y en las precipitaciones.

El aumento en la temperatura de las aguas ha afectado el ciclo de transmisión de algunos parásitos como Fasciola, Fasciolopsis, Opistorchis y Paragonimus (Poulin y Mouritsen, 2006). En el caso de la tripanosomiasis, Pereira et al. (2012) observaron que el aumento en las temperaturas permite 
desarrollar ciclos de vida más cortos y generar mayor densidad de población del parásito.

Según la European Comission (2013), el cambio climático afecta directamente las condiciones de vida de los animales. Estaciones con intensas sequías, calor, frío y humedad excesiva, predisponen al ganado a desarrollar enfermedades bacterianas (Escherichia coli), parasitarias (Fasciola hepática), y trastornos nutricionales como efecto indirecto, asociado con la disponibilidad de alimentos, como el caso de menor crecimiento de praderas por condiciones de sequía (Skuce et al., 2013). Los animales acuáticos como los peces y mariscos, responden directamente a los cambios climáticos, es así como cambios en su entorno generan una mayor susceptibilidad a las ETAs, especialmente en los sistemas intensivos de cultivo (Utaaker y Robertson, 2015).

\section{Globalización}

Los efectos de la globalización en los alimentos, desde la producción de materias primas hasta el consumidor final, son diversos. Durante siglos se ha trasportado ganado desde unas regiones a otras, acompañados de sus respectivas enfermedades (Robertson et al., 2014), muchas de las especies de ganado se han introducido y establecido en países extranjeros, al igual que algunas de sus enfermedades (Jabbar et al., 2014). Una de los mejores ejemplos que se describen relacionado con el transporte de herbívoros es el de Fasciola hepatica, en que la colonización de otros continentes por conquistadores europeos, junto con la introducción de ganado y de equinos proveniente de Europa, además de la adaptación a las especies nativa, ha dado como resultado el establecimiento casi global de este parásito (Mas-Coma et al., 2009). En muchos países se produjo un gran aumento en las exportaciones de cerdos entre los años 1997 a 2007 (Robertson et al., 2014), aumentando simultáneamente los casos de taeniosis, trichinellosis, y toxoplasmosis, por lo que la comercialización de carne de cerdo puede estar asociado con la propagación de estos parásitos, sobre todo si la inspección de carne es precaria (Galán-Puchades y Fuentes, 2014). Meiry et al. (2013) mencionan que la incidencia de cuadros de cisticercosis bovina aumentó de un $4 \%$ al $38 \%$ tras iniciarse la importación de ganado bovino vivo a Israel.

El transporte internacional de animales de compañía, sea por viajes de vacaciones, reubicación de familias, competencias deportivas y movimiento de animales asociados a misiones militares o humanitarias a otras regiones del mundo, representan un riesgo de introducción de agentes patógenos que puedan ser transmitidos por los alimentos, como por ejemplo Echinococcus multi- locularis, complejo Echinococcus granulosus y Toxocara spp. (Macpherson, 2013).

Los animales de vida silvestre son importantes reservorios de patógenos emergentes (Jones et al., 2008); el movimiento de éstos para alimentación, cotos de caza, como reintroducción de especies localmente extintas, para parques zoológicos o simplemente como mascotas, son factores de riesgo (Robertson et al., 2014). En el caso de Trichinella spiralis se han reportado brotes por el consumo de carne de jabalí, de osos y de morsas (Pozio y Zarlenga, 2013).

\section{Comercio de alimentos}

Existen grandes diferencias entre los países exportadores e importadores de productos alimenticios, en las formas de producción, de saneamiento, de higiene, de buenas prácticas agrícolas y en la aparición de agentes patógenos transmitidos por los alimentos (Doyle y Erickson, 2008).

Debido a esto la trazabilidad alimentaria ha tomado importancia, principalmente en los alimentos compuestos, en aquellos que tienen largas cadenas de producción, y en los procesos de producción donde las diversas etapas son llevadas a cabo en diferentes países (Robertson et al., 2014).

Los patógenos que pueden ser transmitidos por la carne son variados. Muchos de ellos están relacionado con el consumo de carne infectada con formas parasitarias de Taenia solium, Taenia saginata, Trichinella spiralis, Toxoplasma gondii, y Sarcocystis spp. (Robertson y Chalmers, 2013). La sarcocistosis, toxoplasmosis, triquinosis y las teniasis, están asociadas con el consumo de carne poco cocida o cruda, que contiene en su interior etapas infectantes de los parásitos. La triquinosis se asocia principalmente con problemas por el faenamiento local de cerdos y no por el comercio internacional de carne (Pozio, 2014). Desde el año 1986 hasta el año 2010, se registraron 65.818 casos de triquinosis en humanos con la muerte de 42 personas reportadas en 41 países (Murrell y Pozio, 2011). La carne de caballo se ha convertido en una fuente importante de infección para Trichinella; dos brotes ocurridos en Francia generaron la infección de 1.073 personas, con resultado de 5 víctimas fatales (Ancelle et al., 1990). La toxoplasmosis representa otro problema; se estima que el 33\% de la población mundial se encuentra infectada con Toxoplasma gondii (Hill y Dubey, 2013). Se ha considerado que el consumo de carne infectada con quistes tisulares y poco cocida es la principal fuente de infección (Cook et al., 2000). Pomares et al. (2011) encontraron que la carne de caballo importada desde Canadá y Brasil estaba implicada en brotes de toxoplasmosis en personas en Francia (Pomares et al., 2011). 
Las tendencias actuales de consumo de carne de fauna exótica ha significado que la carne de caza se está incorporando cada vez más en restaurantes exclusivos, tanto de Europa como de Estados Unidos, pero también la carne de caza ha sido importada para satisfacer las necesidades de las comunidades de inmigrantes de diversos países, generándose así un riesgo alimenticio (Robertson et al., 2014). De este modo Alaria alata en carne de jabalí procedente de Hungría se ha reportado en Francia (Riehn et al., 2010). Spirometra mansoni spargana se han encontrado en ancas de rana importadas desde Indonesia a los Países Bajos (Zhang et al., 2012).

Si bien el consumo de carne exótica no es reciente, este fenómeno ha ido creciendo debido al aumento en el consumo de pescado, en la expansión de la industria acuícola, en las tendencias a consumir alimentos crudos o ligeramente cocidos, y en el escaso desarrollo que ha tenido la utilización de la cadena de frío para el trasporte de los peces a nivel mundial (Chai et al., 2005). Diversas son las especies de parásitos asociados al consumo de carne de pescado; Maestre y Toral (2004) mencionan a Anisakis simplex, Pseudoterranoba dicipiens, Capilllaria philippinensis y Diphyllobothrium latum como las más comunes.

En el caso de las frutas y verduras, los principales agentes infecciosos que transmiten corresponden a parásitos presentes en los productos frescos, lo cual ha dado lugar a brotes de origen alimentario. Por ejemplo, un brote de ciclosporidiosis se presentó por el consumo de vegetales crudos en 73 pasajeros de un crucero que repuso comida procedente de un área endémica de ciclosporidiosis del sudeste asiático (Gibbs et al., 2013).

El potencial de transmisión de parásitos ha aumentado por la moda de consumir productos agrícolas alternativos. El consumo de jugos frescos contaminados con el insecto vector del Trypanosoma cruzi ha dado lugar a brotes de la enfermedad (Pereira et al., 2012); debido a esto el consumo de productos como jugos no pasteurizadas de Acai (Nóbrega et al., 2009), jugos de guayaba, de caña de azúcar y jugo de la baya de palma, podrían ser agentes transmisores de la enfermedad (Toso et al., 2011).

Las especias y hierbas se utilizan en la elaboración de alimentos con la finalidad de mejorar el sabor; la globalización ha hecho que estos productos se encuentren disponibles durante todo el año en un gran número de países, generando brotes alimenticios con una amplia distribución geográfica (Zweifel y Stephan, 2012). Los patógenos contaminantes de especias y hierbas corresponden a aquellos que poseen una mayor resistencia a las condiciones de desecación, como Salmonella y $B a$ cillus cereus (McKee, 1995).

\section{Transporte de pasajeros}

En el año 2010 se estimó que 214 millones de personas ( $3 \%$ de la población mundial) migraron fuera de su país de origen. Esta migración ha resultado también en el movimiento de agentes patógenos transmitidos por los alimentos a diversas regiones del mundo (Robertson et al., 2014).

Entre los patógenos alimentarios que más fácilmente se diseminan entre las personas están los parásitos, muchos de los cuales se encuentran en personas de distintas procedencias, como Taenia saginata (África), Fasciolopsis buski (Asia), Opisthorchis viverrini y Clonorchis sinensis (sudeste asiático), Taenia solium (Sur de Asia), Opisthorchis guayaquilensis (América del Sur), Echinococcus granulosus (Oriente Medio), y Diphyllobotrium spp. y Felineus opisthorchis en Europa del Este (Robertson et al., 2014).

Las personas al viajar llevan sus alimentos $\mathrm{y}$ tradiciones, generando cambios en las preferencias y en los hábitos alimentarios (Broglia y Kapel, 2011). El consumo de carne cruda o poco cocida se asociaba a ciertas culturas y prácticas específicas, lo que se ha ido masificando por la globalización y la modificación de los hábitos alimentarios cosmopolitas (Slifko et al., 2000). Un número creciente de consumidores está buscando productos exóticos (por ejemplo, carne de cocodrilo en Escandinavia), o los productos de temporada durante todo el año, que ahora están fácilmente disponibles en nuestras mesas debido al comercio mundial (por ejemplo, las fresas durante el invierno) (Macpherson, 2005).

\section{DISCUSIÓN}

En esta revisión se abarcaron distintos tema producto de cambios que han estado ocurriendo en las últimas décadas. El conocimiento de los efectos del cambio climático y de la globalización permite generar información que ayude a todos los actores relacionados en la producción de alimentos, en sus distintas etapas o procesos de la producción, a conocer los efectos directos de los cambios anteriormente mencionados en los patógenos zoonóticos que se pueden transmitir por los alimentos, ya sea para disminuir o bien eliminar el riesgo que éstos pudiesen generar sobre la población que los consume. No solo los países importadores de alimentos deben generar medidas precautorias, sino también los países elaboradores/exportadores de alimentos, los cuales deben asegurar alimentos libres de patógenos para las personas.

Se plantean una serie de medidas con la finalidad de reducir estos riesgos. Entre éstas, la comunicación juega un papel importante, ya que permite que los consumidores finales conozcan los 
riesgos asociados a los diversos orígenes de los alimentos, lo que les permite generar las acciones necesarias, como por ejemplo elegir los lugares de adquisición y preparación de los alimentos, para disminuir el riesgo de contraer algún patógeno zoonótico. No solo los consumidores se ven favorecidos con la información disponible; también los productores de alimentos se ven favorecidos al tener acceso a documentación sobre las buenas prácticas de elaboración de alimentos, buenas prácticas ganaderas y puntos críticos de control, entre otros, sino que además de la posibilidad de incorporar nuevas tecnologías en la producción, que les ayudará a generar un alimento seguro e inocuo para la población.

Para llevar a cabo esto, es necesario integrar diversas disciplinas, que aborden cada uno de los elementos que el cambio climático y la globalización están y van a seguir generando sobre los agentes zoonóticos que pudiesen transmitirse a través de los alimentos.

Si bien es cierto que la apertura de mercados, la desaparición de las barreras comerciales entre países, y la facilidad de desplazamiento de personas de un lugar a otro del planeta, han generado beneficios, no se pueden desconocer los riesgos que estos generan en la transmisión de enfermedades desde una región a otra. Es ahí donde las diversas organizaciones, tanto nacionales como internacionales, relacionadas con la producción de alimentos, con la sanidad animal y con la salud humana, deben velar de forma constante para identificar los riesgos y el impacto que las zoonosis transmitidas por los alimentos tienen sobre la población, y generar las medidas de control y de prevención tendientes a disminuir, o si fuese posible, eliminar los riesgos que los alimentos pueden generar sobre la población.

Debido a que los procesos relacionados con el cambio climático y globalización son continuos y dinámicos, las investigaciones se deben seguir llevando a cabo, ya que existe incertidumbre sobre como a futuro los cambios mencionados afectarán la presentación de estas enfermedades.

\section{CONCLUSIONES}

El cambio climático puede afectar la incidencia de las ETAs a través de su efecto sobre los patógenos, hospedadores y ambiente. Estos cambios pueden afectar las rutas migratorias de animales silvestres, generando la aparición de ciertos patógenos y la desaparición de otros en determinadas zonas.

El comercio internacional y el movimiento de personas son puntos clave en el proceso de globalización. El contacto de las personas con otras culturas, costumbres, tradiciones y la adopción de estas, representa un riesgo de contraer ETAs.

El movimiento de animales, ya sea de forma legal o de contrabando, pueden significar la diseminación de enfermedades de una región del planeta a otra.

Con la finalidad de definir el efecto del cambio climático y de la globalización sobre el comportamiento epidemiológico de las ETAs es necesario fortalecer los elementos de los sistemas de control de los alimentos: coordinación y gestión; legislación, vigilancia, supervisión de los servicios de laboratorio y de inspección; educación, información y comunicación.

\section{LITERATURA CITADA}

Altizer S., R.S. Ostfeld, P.T. Johnson, S. Kutz, and C.D. Harvell. 2013. Climate change and infectious diseases: from evidence to a predictive framework. Science 341(6145):514-9. doi: 10.1126/science.1239401.

Ancelle T., G. Renaud, J. Dupouy-Camet, and G. Foulon. 1990. Evaluation of the medical and social cost of 2 trichinosis outbreaks in France in 1985. Rev. Epidemiol. Sante Publique 38:179-186.

Broglia A., and C. Kapel. 2011. Changing dietary habits in a changing world: Emerging drivers for the transmission of foodborne parasitic zoonoses. Veterinary Parasitology 182:2-13.

Chai J-Y., K.D. Murrell, and A.J. Lymbery. 2005. Fish-borne parasitic zoonoses: Status and issues. International Journal for Parasitology 35:1233-1254.

Cook A.J., R.E. Gilbert, W. Buffolano, J. Zufferey, E. Petersen, P.A. Jenum, et al. 2000. Sources of Toxoplasma infection in pregnant women: European multicentre case-control study. Br. Med. J. 321:142-147.

Cullen, E. 2009. The impact of climate change on the future incidence of specified foodborne diseases in Ireland. In Conference of the International Society of Environmental Epidemiologists. Lippincott Williams \& Wilkins, Inc., Dublin, Ireland.

Doyle, M.P., and M.C. Erickson. 2008. Imported Foods. Microbiological Issues and Challenges. ASM Press, Georgia, USA.

Drake, S. L., A. DePaola, and A. Jaykus. 2007. An overview of Vibrio vulnificus and Vibrio parahaemolyticus. Comprehensive Reviews in Food Science and Food Safety 6:120-144.

European Commission. 2013. Adaptation to climate change impacts on human, animal and plant health. Commission Staff Working Document. Brussels, 16.4.2013 SWD (2013) 136 final. 
Friedman C.R., R.M. Hoekstra, M. Samuel, R. Marcus, J. Bender, B. Shiferaw, et al. 2004. Risk factors for sporadic Campylobacter infection in the United States: A case-control study in Food Net sites. Clin Infect Dis. 15(38):285-296.

Galán-Puchades, M.T., and M.V. Fuentes. 2014. Parasitic porkborne hazards, globalisation, and meat inspection. Food Control 46:546-547.

Gibbs R.A., R. Nanyonjo, N.M. Pingault, B.G. Combs, T. Mazzucchelli, P. Armstrong, et al., 2013. An outbreak of Cyclospora infection on a cruise ship. Epidemiol. Infect. 141:508-516.

Gregory P.J., J.S.I Ingram, and M. Brklacich. 2005. Climate change and food security. Phil. Trans. R. Soc. B 360:2139-2148.

Hashizume, M., Y. Wagatsuma, A.S.G. Faruque, H. Taiichi Hayashi, and B. Armstrong. 2009. Climatic components of seasonal variation in cholera incidence. In Conference of the International Society of Environmental Epidemiologists. Lippincott Williams \& Wilkins Inc., Dublin, Ireland.

Hill, D.E., and J.P. Dubey. 2013. Toxoplasma gondii prevalence in farm animals in the United States. Int. J. Parasitol. 43:107-113.

Jabbar A., J. Cotter, J. Lyon, A.V. Koehler, R.B. Gasser, and B. Besier. 2014. Unexpected occurrence of Haemonchus placei in cattle in southern Western Australia. Infect. Genet. Evol. 21:252-258.

Jones K.E., N.G. Patel, M. A. Levy, A. Storeygard, D. Balk, J. L. Gittleman, and P. Daszak. 2008. Global trends in emerging infectious diseases. Nature 451:990-993.

Kovats R.S., S.J. Edwards, D. Charron, J. Cowden, R.M. D'Souza, K.L. Ebi, et al., 2005. Climate variability and campylobacter infection: An international study. International Journal of Biometeorology 49:207-214.

Macpherson, C.N. 2013. The epidemiology and public health importance of toxocariasis: a zoonosis of global importance. Int. J. Parasitol. 43(12-13):999-1008.

Maestre Vera J.R., y J.R. Toral. 2004. Parasitosis intestinal por nematodos asociada al consumo de pescado contaminado. Rev. Clin. Esp. 204(5):264-5.

Marques A., M.L. Nunes, S.K. Moore, M.S. Stromb. 2010. Climate change and seafood safety: Human health implications. Food Research International. 43(7):1766-1779.

Mas-Coma S., M.A. Valero, and M.D. Bargues. 2009. Fasciola, lymnaeids and human fascioliasis, with a global overview on disease transmission, epidemiology, evolutionary genetics, molecular epidemiology and control. Adv. Parasitol. 69:41-146.
McKee, L.H. 1995. Microbial contamination of spices and herbs: A review. LWT - Food Science and Technology 28(1):1-11.

Meiry M., G. Brenner, A. Markovitcs, and E. Klement. 2013. A change in the epidemiology of bovine cysticercosis in Israel between 1973 and 2008 due to import of live cattle. Transbound. Emerg. Dis. 60:298-302.

Millán, M., 2014. Extreme hydrometeorological events and climate change predictions in Europe. Journal of Hydrology 518(Part B):206-224.

Murrell, K.D. and E. Pozio. 2011. Worldwide occurrence and impact of human trichinellosis, 1986-2009. Emerg. Infect. Dis. 17:2194- 2202.

Nóbrega, A.A., M.H. Garcia, E. Tatto, M.T. Obara, E. Costa, J. Sobel, et al. 2009. Oral transmission of Chagas disease by consumption of açaí palm fruit, Brazil. Emerg. Infect. Dis. 15(4):653-655.

OMS. 2007. Informe sobre la salud en el mundo. Protección de la salud pública mundial en el siglo XXI: un porvenir más seguro. Organización Mundial de la Salud (OMS). Disponible en http://www.who.int/whr/2007/07_ report_es.pdf?ua $=1$ (Consulta 14 abril de 2015).

Pereira K.S., R.L. Barbosa, L.A.C. Passos, F.S. de Aguiar, H. Rogez, B. Alarcón de Noya, et al. 2012. Trypanosoma cruzi. p. 189-216. In H.V. Smith, L.J. Robertson (eds.) Foodborne Protozoan Parasites. Nova Publishers, New York, USA.

Polley, L., S.J. Kutz, and E.P. Hoberg. 2011. Parasite Zoonoses. p. 325-345. In I.O. Nriagu, N. (ed.). Encyclopedia of Environmental Health, Five Volume Set. 4:325-345 (invited chapter). Elsevier Science, London, U.K.

Pomares C., D. Ajzenberg, L. Bornard, G. Bernardin, L. Hasseine, M.L. Darde, and P. Marty. 2011. Toxoplasmosis and horse meat, France. Emerg. Infect. Dis. 17:1327-1328.

Poulin, R., and K.N. Mouritsen. 2006. Climate change, parasitism and the structure of intertidal ecosystems. Journal of Helminthology 80:183-191.

Pozio E., and D.S. Zarlenga. 2013. New pieces of the Trichinella puzzle. International Journal for Parasitology 43(12-13):983-997.

Pozio, E. 2014. Searching for Trichinella: not all pigs are created equal. Trends Parasitol. 30(1):4-11.

Riehn K., A. Hamedy, K. Grosse, L. Zeitler, and E. Lücker. 2010. A novel detection method for Alaria alata mesocercariae in meat. Parasitol. Res. 107:213-220. 
Robertson L.J., H. Sprong, Y.R. Ortega, J.W.B. van der Giessen, and R. Fayer. 2014. Impacts of globalization on foodborne parasites. Trends in Parasitology 30(1):37-52.

Robertson, L.J., and R.M. Chalmers. 2013. Foodborne cryptosporidiosis: is there really more in Nordic countries? Trends Parasitol. 29:3-9.

Skuce P.J., E.R. Morgan, J. van Dike, and M. Mitchell. 2013. Animal health aspects of adaptation to climate change: beating the heat and parasites in a warming Europe. Animal 7(Suppl 2):333-345. doi: 10.1017/S175173111300075X.

Slifko T.R., H.V. Smith, and J.B. Rose. 2000. Emerging parasite zoonoses associated with water and food. Int. J. Parasitol. 30(1213):1379-93

Tirado M.C., R. Clarke, L.A. Jaykus, A. McQuatters-Gollop, and J.M. Frank. 2010. Climate change and food safety: A review. Food Research International 43:1745-1765.

Toso A., F. Vial, and N. Galanti. 2011. Oral transmission of Chagas' disease. Rev. Med. Chil. 139:258-266.
Utaaker K.S., and L.J. Robertson. 2015. Climate change and foodborne transmission of parasites: A consideration of possible interactions and impacts for selected parasites. Food Research International 68:16-23.

Uyttendaele M., C.Liub, and N. Hofstra. 2015. Special issue on the impacts of climate change on food safety. Food Research International. 68,1-6

Zhang X.P., S.F. Jiang, G.B. Hong, Y.H. Fu, Y.Y $\mathrm{He}$ X.J. Ma, et al., 2012. Investigation on food contamination with parasites in Shanghai market. Zhongguo Xue Xi Chong Bing Fang Zhi Za Zhi. 24:404-409

Zweifel C., and R. Stephan. 2012. Spices and herbs as source of Salmonella-related foodborne diseases. Food Research International 45:765-769. 